\title{
Une méthode simple pour maintenir et multiplier les champignons des mycorhizes à vésicules et arbuscules
}

\author{
K Boudarga, J Dexheimer \\ Université de Nancy I, laboratoire de biologie des ligneux, BP 239, 54506 Vandœuvre-lès-Nancy cedex, France
}

(Reçu le 3 juin 1989; accepté le 6 mars 1990)

\begin{abstract}
Résumé - Les auteurs présentent une méthode simple pour maintenir et multiplier les champignons des mycorhizes VA, utilisant comme plante hôte des boutures de Plectranthus australis. Les plantes sont cultivées, dans des bocaux fermés, sur un substrat composé d'un mélange de tourbe et de vermiculite. Dans ces conditions de culture, les plantes hôtes présentent une bonne mycorhization. Des fragments du système racinaire ou du substrat de culture contenant des spores et des sporocarpes de l'endophyte peuvent être utilisés comme inoculum. La culture en milieu clos et l'utilisation de racines néoformées, n'ayant jamais été en contact avec la microflore du sol, limitent considérablement les risques d'infection accidentelle.
\end{abstract}

mycorhize à vésicules et arbuscules / Plectranthus australis / bouture / inoculum

Summary - A simple method for the culture and the multiplication of VA mycorrhizal fungus. The authors present a simple method for the culture and the multiplication of VA mycorrhizal fungus on Plectranthus australis cuttings (fig 1). The host-plant is easily propagated by cuttings, ensuring the production of a large number of new adventitious roots which have never been in contact with the soil microflora (fig 2). The plants are cultivated in closed jars on a substrate composed of a mixture of peat and vermiculite (fig 3). Examination by light and electron microscopy has shown that a Plectranthus culture in a confined environment does not have a reduced mycorrhization capacity and that the mycorrhiza formed present the same organisation as those observed under natural or artificial conditions (figs 4, 6, 7). Fragments of the root system or culture substrate containing hyphae, spores and sporocarps of the endophyte (fig 5) may be used as inoculum for the multiplication of the fungus.

vesicular-arbuscular mycorrhiza / Plectranthus australis / cutting / inoculum / controlled mycorrhization

\section{INTRODUCTION}

L'utilisation pratique des mycorhizes passe par des études théoriques sur les modalités de leur établissement, de leur fonctionnement et de leur maintien sur la plante hôte.

Pour ces études, il est souvent nécessaire de pouvoir disposer de mycorhizes obtenues en conditions contrôlées, ce qui implique la capacité de contrôler la culture des 2 partenaires, notamment du symbiote fongique, afin de réaliser les inoculations.

En général, la culture des champignons des symbioses ectomycorhiziennes est relativement facile sur des milieux artificiels et diverses tech- niques efficaces de production d'inoculum ont été mises au point (Le Tacon et al, 1983; Mauperin et al, 1987).

De même, le champignon responsable de la formation des mycorhizes éricoïdes (Pezizella ericae) peut être aisément maintenu sur un milieu synthétique. Son aptitude à se segmenter en courts fragments est particulièrement favorable à la production d'inoculum (Pons et al, 1982).

Le problème est beaucoup plus complexe avec les champignons des mycorhizes à vésicules et arbuscules. En effet, les Endogonacées sont des symbiotes obligatoires qui doivent toujours être associés avec une plante hôte et, malgré de nombreux essais (Strullu et Romand, $1986,1987)$, on ne sait pas cultiver ces champi- 
gnons sur un milieu synthétique. Le problème est d'autant plus aigu que ce type de mycorhize est largement répandu et qu'il est rencontré aussi bien dans des plantes herbacées d'intérêt agronomique que dans des essences forestières tempérées ou tropicales.

Plusieurs méthodes de production d'inoculum ont été proposées (Biermann et Linderman, 1983; Gonzalez et Barrios, 1983; Elmes et Mosse, 1984; Mosse et Thompson, 1984; Elmes et al, 1984; Dehne et Backhaus, 1986; Mugnier et Mosse, 1987; Coltman et al, 1988; Hung et Sylvia, 1988; Sreenivasa et Bagyaraj, 1988a et b). Compte tenu des difficultés de culture des Endogonacées, le principe de la plupart de ces méthodes est de réaliser une culture préalable sur une plante hôte intermédiaire, parfois réduite à un système racinaire transformé (Mugnier et Mosse, 1987) et d'utiliser des fragments de racines infectées ou du substrat de culture comme inoculum. Beaucoup plus rarement, ce sont les vésicules qui constituent l'inoculum (Biermann et Lindermann, 1983; Strullu et Romand, 1987).

Ces techniques diffèrent par le choix de la plante hôte et par sa culture, effectuée, soit en pot, soit en culture hydroponique ou aéroponique, en conditions axéniques ou non.

Beaucoup de ces techniques nécessitent une mise en œuvre complexe et un équipement sophistiqué et coûteux. II nous est donc apparu intéressant de présenter les résultats préliminaires d'une nouvelle méthode simple permettant de maintenir le champignon et d'obtenir de l'inoculum en milieu clos.

\section{MATÉRIEL ET MÉTHODES}

\section{Choix de la plante hôte}

Nous avons retenu comme plante hôte, une espèce herbacée de la famille des Labiacées, d'origine australienne, le Plectranthus australis (fig 1). II s'agit d'une espèce peu exigeante, présentant une croissance continue toute l'année et offrant une grande aptitude au bouturage et à la production d'un chevelu de fines racines.

\section{Choix des endophytes}

Pour les mycorhizations, nous avons utilisé plusieurs espèces d'Endogonacées :

- Glomus mosseae,
- Glomus intraradices,

- Glomus monosporus,

- Gigaspora margarita.

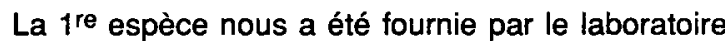
de microbiologie du centre de recherches forestières de l'INRA, à Champenoux, les 3 autres espèces par le laboratoire de physiopathologie végétale de l'INRA à Dijon.

Avant leur transfert sur Plectranthus australis, ces espèces sont maintenues sur de l'oignon ou du poireau.

\section{Culture et mycorhization de Plectranthus australis en conditions contrôlées}

La culture et la mycorhization de Plectranthus australis sont effectuées dans des bocaux de 11 à couvercle métallique vissé.

Les bocaux sont remplis à moitié d'un substrat composé de $2 / 3$ de vermiculite et $1 / 3$ de tourbe non fertilisée, et imprégné par $100 \mathrm{~cm}^{3}$ du milieu de MelinNorkrans, modifié par Marx (1969), et dans lequel nous avons réduit de moitié la quantité de glucose.

$\begin{array}{lc}\text { - }\left(\mathrm{NH}_{2}\right)_{2} \mathrm{HPO}_{4} & 1,89 \mathrm{~mol} . \mathrm{I}^{-1} \\ -\mathrm{MgSO}_{4}, 7 \mathrm{H}_{2} \mathrm{O} & 0,61 \mathrm{~mol} . \mathrm{I}^{-1} \\ -\mathrm{KH}_{2} \mathrm{PO}_{4} & 0,37 \mathrm{~mol} . \mathrm{I}^{-1} \\ -\mathrm{NaCl} & 0,43 \mathrm{~mol} . \mathrm{I}^{-1} \\ -\mathrm{CaCl}_{2} & 0,49 \mathrm{~mol} . \mathrm{I}^{-1} \\ \text { - thiamine } \mathrm{HCl} & 0,29 \mathrm{~mol} . \mathrm{I}^{-1} \\ \text { - glucose } & 27,8 \mathrm{~mol} . \mathrm{I}^{-1} \\ \text { - extrait de malt } & 3,0 \mathrm{~g} \\ \text { - citrate ferrique à 1\% } & 1,2 \mathrm{ml} \\ \text { - eau distillée } & 1000 \mathrm{ml}\end{array}$

Les bocaux renfermant le substrat et le milieu nutritif sont désinfectés par un passage à l'autoclave durant $20 \mathrm{~min}$ à $120^{\circ} \mathrm{C}$.

Les fragments de tige de Plectranthus australis, comprenant 1 ou 2 nœuds avec des feuilles bien développées et un entre-nœud, sont désinfectés par un trempage de $1 \mathrm{~min}$ dans l'alcool absolu puis rincés plusieurs fois par l'eau distillée stérile, ils sont ensuite légèrement enfoncés $(2$ à $3 \mathrm{~cm}$ ) dans le substrat de culture contenu dans les bocaux.

L'inoculum introduit en même temps que la bouture peut être constitué, soit par des fragments de racines d'une plante mycorhizée et qui sont désinfectés comme les boutures, soit pour Gigaspora margarita, par des spores désinfectées en surface (Pons, 1984).

Toutes les opérations sont effectuées sous la hotte à flux laminaire.

Les bocaux sont ensuite fermés et placés sous une rampe lumineuse (12 tubes Sylvania Grolux de $30 \mathrm{~W}$ ) réglée en jours de $12 \mathrm{~h}$. La température est comprise entre 25 et $28^{\circ} \mathrm{C}$. 
Les témoins sont réalisés en cultivant des boutures en bocal sur le substrat désinfecté auquel il n'a pas été rajouté d'inoculum.

\section{Techniques d'observation}

\section{Microscope photonique}

\section{Coloration par le bleu trypan}

Après 2 mois de culture, et pour contrôler l'installation de la mycorhize, nous avons prélevé des racines et pratiqué une coloration selon la méthode de Phillips et Hayman (1970).

\section{Observation des coupes semi-minces}

Les coupes semi-minces sont réalisées à partir d'objets préparés pour la microscopie électronique. Elles sont colorées par le bleu de toluidine à pH alcalin.

\section{Microscopie électronique}

Les fragments de racine ont été fixés par la glutaraldéhyde à $2,5 \%$ dans du tampon phosphate à $\mathrm{pH} 7,2$ pendant 6 à $8 \mathrm{~h}$ à la température de la glace fondante, ensuite les objets sont rincés par le même tampon, puis postfixés à l'osmium $(2 \%, 1 \mathrm{~h})$, déshydratés par l'acétone et inclus dans l'épon (Boudarga et Dexheimer, 1988; Boudarga, 1989).

Les coupes minces sont faites à l'ultramicrotome à l'aide d'un couteau de diamant. Elles sont recueillies sur des grilles en cuivre puis colorées par le citrate de plomb et l'acétate d'uranyle.

\section{RÉSULTATS}

\section{Choix des boutures}

Pour déterminer quel type de bouture assure une production rapide et importante de racines, nous avons bouturé des fragments de plantes comprenant :

-1 nœud avec une paire de feuilles et un entrenœud,

- 2 nœuds feuillés séparés par un entre-nœud,

-1 ou 2 nœuds avec ablation des feuilles.

Dans tous les cas, il se forme, le long des entre-nœuds, des racines adventives. Les bourgeons axillaires se développent et donnent une tige feuillée (fig 2). Toutefois, les boutures comprenant 2 nœuds feuillés présentent la croissance la plus rapide et le développement racinaire le plus important. C'est ce type de bouture que nous avons utilisé pour la suite des expériences.

En une dizaine de j, le substrat est colonisé par les racines qui sont bien visibles à travers la paroi du bocal (fig 3).

\section{Contrôle de la mycorhization}

Deux mois après l'introduction de l'inoculum (racines infectées ou spores) dans le bocal, nous avons pratiqué des contrôles en microscopie photonique et électronique, afin d'apprécier le degré d'infection des boutures de Plectranthus australis. Pour toutes les espèces d'endophyte, les résultats sont identiques à quelques détails près, comme l'abondance relative des arbuscules et des vésicules.

\section{Coloration par le bleu trypan}

Dans les racines colorées par le bleu trypan, nous avons observé un réseau d'hyphes intercellulaires et des arbuscules. Cependant, certaines racines contiennent surtout des vésicules inter- et intracellulaires. L'infection est très forte et quasiment toutes les racines observées présentent une colonisation importante par l'endophyte (fig 4).

Les racines des témoins sont toujours indemnes d'infection.

\section{Coupes semi-minces}

L'observation, après coloration par le bleu de toluidine, de coupes semi-minces montre que les arbuscules sont localisés dans les cellules du cortex interne de la racine (fig 6). Le réseau d'hyphes intercellulaires pouvant être, soit très dense, soit beaucoup plus lâche.

\section{Microscopie électronique}

L'examen au microscope électronique de coupes minces permet de préciser ces observations. Les cellules du cortex interne dont le cytoplasme est dense et peu vacuolisé renferment de nombreux hyphes, de diamètres variés correspondant à un arbuscule (fig 7). L'interface entre les hyphes et le cytoplasme de la cellule hôte est toujours limitée par une membrane plasmalemmique.

De même, dans les méats intercellulaires, des sections d'hyphes de gros diamètre témoignent de l'existence d'un réseau intercellulaire. 
Cette organisation est celle qui a déjà été décrite de nombreuses fois dans les mycorhizes à vésicules et arbuscules naturelles ou artificielles.

\section{Examen du substrat de culture}

Le substrat de culture a été tamisé selon la technique préconisée par Gerdemann et Nicolson (1963) et, en examinant sous la loupe binoculaire ou le microscope les diverses fractions, nous avons observé de nombreux sporocarpes et les spores de l'endophyte (fig 5).

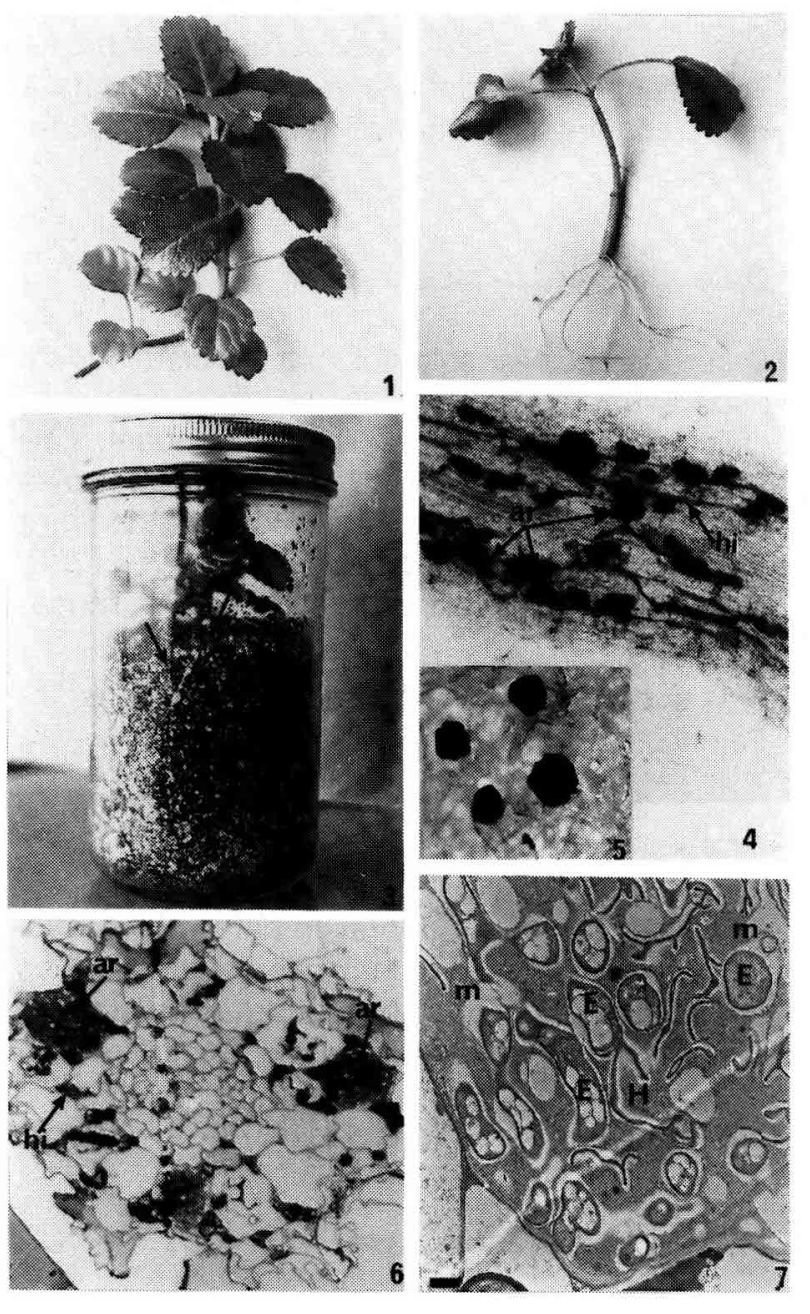

Fig 1. Fragment de tige feuillée de Plectranthus australis. Fig 2. Une bouture enracinée.

Fig 3. Culture des boutures en bocal de verre sur un mélange de tourbe et de vermiculite. Remarquer (flèches) les racines appliquées sur la paroi de verre.

Fig 4. Coloration par le bleu trypan d'une racine révélant la présence d'un réseau d'hyphes intercellulaires et d'arbuscules (G X 180).

Fig 5. Sporocarpes de Glomus mosseae, isolés à partir du substrat ( $G \times 253$ ).

Fig 6. Coupe semi-mince d'une racine hébergeant l'endophyte ( $\mathrm{G} \times 676$ ).

Fig 7. Microphotographie électronique d'une cellule hôte renfermant de nombreuses sections d'un arbuscule ( $G \times 3900)$. La longueur du trait noir représente $1 \mu \mathrm{m}$.

\section{Utilisation des racines et du substrat comme inoculum}

Enfin, nous avons réalisé des infections en conditions contrôlées en utilisant comme inoculum des fragments de racine de Plectranthus australis, du substrat de culture ou, pour Gigaspora margarita, des spores isolées par tamisage (Gerdemann et Nicolson, 1963).

L'inoculum a été essayé sur des boutures de Plectranthus australis et sur des plantules d'Eucalyptus camaldulensis obtenues, soit par germination de graines, soit par micropropagation. L'infection a été réalisée en bocal sur un substrat stérile ou pour les spores isolées, en boîtes de Petri selon la technique que nous avons précédemment décrite (Boudarga et Dexheimer, 1988).

Dans tous les cas et quelles que soient la nature de l'inoculum et la technique de mycorhization employée, en 8 semaines, nous avons observé la formation d'endomycorhizes à vésicules et arbuscules.

\section{DISCUSSION ET CONCLUSION}

La taille importante de la plupart des plantes hôtes utilisées exclut la possibilité d'obtenir leur développement en milieu confiné, condition importante pour limiter les infections accidentelles. II est d'ailleurs difficile d'obtenir des plantes hôtes parfaitement axéniques, sauf si l'on utilise des racines transformées ou des graines qui peuvent être désinfectées en surface. Mais il faut alors attendre plusieurs semaines pour obtenir une quantité importante de racines.

Le Plectranthus australis présente une grande aptitude au bouturage, ce qui garantit une production rapide de racines adventives néoformées qui, n'ayant jamais été en contact avec la microflore du sol, sont indemnes de toute infection. Les problèmes de désinfection de la plante hôte sont donc très simplifiés, puisqu'il suffit de désinfecter la bouture sans racines, avant l'introduction dans le bocal de culture.

Bien que la plante puisse présenter un grand développement (tiges de plusieurs dizaines de $\mathrm{cm}$ ), il est tout à fait possible de la maintenir en bocal pendant plusieurs semaines.

Nos études antérieures (Boudarga, 1989) ont montré que cette espèce peut très facilement et très abondamment former des mycorhizes avec les Endogonacées. 
Les vérifications par observation en microscopie photonique et électronique ont montré que la culture des Plectranthus en milieu confiné ne modifie pas l'aptitude à la mycorhization et que les mycorhizes formées présentent une organisation identique à celle observée sur des mycorhizes obtenues avec des plantes cultivées en pots (Boudarga, 1989).

Enfin, des fragments de racines renfermant l'endophyte ou du substrat contenant des hyphes, des spores et des sporocarpes, peuvent être utilisés comme inoculum pour multiplier le champignon. Nous avons ainsi infecté des boutures de Plectranthus australis, des semis et des vitroplants d'Eucalyptus camaldulensis.

L'utilisation de boutures de Plectranthus australis élevées en milieu confiné, en conditions axéniques, apparaît donc comme tout à fait valable pour maintenir en conditions contrôlées les champignons endomycorhizogènes et produire de l'inoculum.

II est vraisemblable que cette technique puisse aussi être utilisée avec d'autres espèces de plante hôte se bouturant facilement et présentant un développement réduit, compatible avec la culture en milieu confiné. Cependant, nous ne l'avons développée actuellement qu'à une petite échelle, pour maintenir nos souches et produire l'inoculum nécessaire aux travaux du laboratoire. II est certain que d'autres études sont nécessaires pour passer à une production plus importante d'inoculum.

\section{RÉFÉRENCES}

Biermann B, Linderman RG (1983) Use of vesiculararbuscular mycorrhizal roots, intraradical vesicles and extraradical vesicles as inoculum. New Phytol 95, 97-105

Boudarga K (1989) Étude des mycorhizes de l'Eucalyptus camaldulensis. Application pratique à la mycorhization de vitroplants. Thèse Univ Nancy I

Boudarga K, Dexheimer J (1988) Étude ultrastructurale des endomycorhizes à vésicules et arbuscules de jeunes plants d'Eucalyptus camaldulensis (Dehnardt) (Myrtacées). Bull Soc Bot Fr 135, 2, 111-121

Coltmann RR, Waterer DR, Huang RS (1988) A simple method for production of Glomus aggregatum inoculum using controlled-release fertilizer. Hortsci 23 , 213-215

Dehne HW, Backhaus GF (1986) The use of vesiculararbuscular mycorrhizal fungi in plant production. I: Inoculum production. $Z$ Pflanzenkr Pflanzenschutz $93,415-424$
Elmes RP, Mosse B (1984) Vesicular-arbuscular endomycorrhizal inoculum production. II: Experiments with maize (Zea mays) and other hosts in nutrient flow culture. Can J Bot 62, 1531-1536

Elmes RP, Hepper CM, Hayman DS, Osheau J (1984) The use of vesicular-arbuscular mycorrhizal roots grown by the nutrient film technique as inoculum for field sites. Ann Appl Biol 104, 437-441

Gerdemann JW, Nicolson TH (1963) Spores of mycorrhizal Endogone species extracted from soil by wet sieving and decanting. Trans Br Mycol Soc 46, 234-235

Gonzalez S, Barrios SRA (1983) Produccion de inoculo de mycorrizas arbusculares. Rev Latinoam Microbiol 25, 181-187

Hung LLL, Sylvia DM (1988) Production of vesiculararbuscular mycorrhizal fungus inoculum in aeroponic culture. Appl Environ Microbiol 54, 353-357

Le Tacon F, Jung G, Michelot P, Mugnier M (1983) Efficacité en pépinière forestière d'un inoculum de champignon ectomycorhizien produit en fermenteur et inclus dans une matrice de polymères. Ann Sci For 40, 165-176

Marx DH (1969) The influence of ectotrophic mycorrhizal fungi on the resistance of pine roots to pathogenic infection. I. Antagonism of mycorrhizal fungi to root pathogenic fungi and soil bacteria. Phytopathology 59, 153-163

Mauperin C, Mortier F, Garbaye J, Le Tacon F, Carr $G$ (1987) Viability of an ectomycorrhizal inoculum produced in a liquid medium and entrapped in a calcium alginate gel. Can J Bot 65, 2326-2329

Mosse B, Thompson JP (1984) Vesicular-arbuscular endomycorrhizal inoculum production. I: Exploratory experiments with beans (Phaseolus vulgaris) in nutrient flow culture. Can J Bot 62, 1523-1530

Mugnier J, Mosse B (1987) Vesicular-arbuscular mycorrhizal infection in transformed root-inducing $T$ DNA roots grown axenically. Phytopathology 77 , 1045-1050

Phillips JM, Hayman DS (1970) Improved procedures for clearing roots and staining parasitic and VA mycorrhizal fungi for rapid assessement of infection. Trans Br Mycol Soc 55, 158-161

Pons F (1984) L'endomycorhization VA in vitro de quelques espèces herbacées et ligneuses; aspects ultrastructuraux de lassociation. Thèse de IIIe cycle. Univ Dijon, 116 pp

Pons, Gianinazzi-Pearson V, Gianinazzi S (1982) Synthèse in vitro des endomycorhizes ericoïdes et VA : complément à la vitropropagation. Colloq INRA, 13, 345-349

Sreenivasa MN, Bagyaraj DJ (1988a) Chloris gayana (Rhodes grass), a better host for the mass production of Glomus fasciculatum inoculum. Plant Soil 106, 289-290

Sreenivasa MN, Bagyaraj DJ (1988b) Optimum frequency of addition of nutrient solution for mass production of Glomus fasciculatum inoculum. Curr Sci $57,665-667$ 
Strullu DG, Romand C (1986) Méthode d'obtention d'endomycorhizes à vésicules et arbuscules en conditions axéniques. CR Acad Sci Paris, 303, 245-250
Strullu DG, Romand C (1987) Culture axénique de vésicules isolées à partir d'endomycorhizes et réassociation in vitro à des racines de tomates. $C R$ Acad Sci Paris 305, 15-19 